\title{
Implementation of Biography Based Neural Clustering (BBNC) with Genetic Processing for tumor detection from medical images
}

\author{
Chandanpreet kaur ${ }^{1}$ and Bikrampal kaur ${ }^{2}$ \\ ${ }^{1} M$ tech student (IT Department), Chandigarh Engineering College, Landran , Mohali, Punjab, India \\ ${ }^{2}$ Professor (CSE Department), Chandigarh Engineering College, Landran, Mohali, Punjab, India
}

\begin{abstract}
Segmentation is a best method to divide the required region from the medical images. This research is based on segmentation of medical images (MRI, CT scans) based on the previous method known as pre-operative and post-recurrence tumor registration (PORTR) and proposed method biography based neural clustering (BBNC) with genetic processing for tumor segmentation. By using the new technique the extracted part can be view in 3D model and also can get the actual segmented tumor region. This new method will be helpful for diagnostics to find the tumor area as well as pixel difference in segmented part to define the tumor area accurately. While in the previous approach all the parameters have been used likewise, in which the registration method is used to transform the different sets of data into one coordinate system for segmentation of medical images. Registration basically is used to improve the signals to reduce the noise from the images. These techniques are better to find the tumor area from the MRI and CT scans, but after comparing them better results have been obtained in proposed technique. The proposed technique (BBNC) reduces the extracted region again into required and actual region of tumor with accuracy of area, time and pixel difference.
\end{abstract}

\section{INTRODUCTION}

Brain tumor is defined as the irregular development of the cells from another part of body to brain or in the brain. There are two types of tumors the first one is known as primary brain tumor and second one is known as secondary brain tumor. Primary brain tumors are start from the brain and the secondary brain tumors are the consequence of melanoma. Percentage of brain tumors is $7-9 \%$ of all cancers. Approximately $20 \%-40 \%$ of brain tumors are due to variations from the norm in the body and can strike at any age [9]. The advancements of imaging strategies, for example, Computer Tomography (CT) and Magnetic Resonance Imaging (MRI) offer specialists with very high resolution images, which will enormously helped in the clinical diagnosis. In the mean time medical experts need to process countless with a great deal more subtle elements. Magnetic resonance imaging (MRI) is a progressed diagnostic tool that helps diagnostics to picture anatomical subtle elements more plainly and so prevalent in identifying variations from the norm in the soft tissues of the brain. Therefore Segmentation techniques have been used for this purpose which is normally a long and decisive process commonly used from long time [7].This research will presents the comparison between previous method (PORTR) and proposed method (BBNC) to extract the tumor region from the medical images, and also will presents the 3-D view of extracted region from the medical images. There are various image segmentation procedures and they are discussed below:

\section{Threshold Based Segmentation}

Histogram thresholds are utilized to fragment the particular image. There are certain pre-processing and post-processing strategies are required to threshold the images [10]. The Major Thresholding procedures are proposed by diverse scientists i.e. Mean Strategy, P-Tile system, visual system, Edge Maximization strategy, and Histogram subordinate method Histogram subordinate method.

Thresholding is one of the oldest method to segment the different type of images. The segmentation can be done by making all the pixels together in one class. On the identification of a good threshold, this strategy depends and failing of such threshold may guide to poor segmentation. A procedure to determinate more than one threshold value is called multi-thresholding. In a sequence of image processing operations thresholding is utilized as an initial step.

\section{Region Based Segmentation}

\footnotetext{
${ }^{\mathrm{a}}$ Corresponding author: $\underline{\text { Chandangill4@gmail.com }}$
} 
Region based segmentation isolates an image into diverse areas in view of pre-characterized criteria, i.e., colour, intensity, or object [11]. Region based segmentation strategies are sorted into three primary classes, i.e. region growing, region splitting, and region merging. For image segmentation region growing strategy is a well developed procedure. Taking into account some predefined criteria this technique extracts image region. This depends on intensity information or edges in the image. An operator manually selects a seed point and extracts all pixels that are associated to the initial seed based on some predefined criteria. An algorithm for this type of segmentation is called as split-and-merge algorithm which is identified with region growing algorithm.

\section{ANN Based Segmentation}

The method for segmentation can be Artificial or we can say men made so the Artificial Neural Network. In artificial neural network every neuron is relating to a particular pixel of a picture, then picture is mapped to the neural system.. In neural system the picture is prepared by utilizing the training samples and afterward association between neurons when pixels are found. At that point the new pictures are fragmented from the trained image [12].

Neural network based segmentation have four essential attributes:

(i) It has parallel work capacity and quick registration ability, which makes it suitable for real time application.

(ii) Improve the segmentation results when the data deviates from the normal situation.

(iii) The robustness making it insensitive to noise.

(iv) It reduces the requirement of experts' intervention for image segmentation process.

\section{PDE Based Segmentation}

The Partial Differential equations or PDE models are utilized generally as a part of image processing and particularly in image segmentation. These equations are active contour model for segmentation purpose. Active Contour model or Snakes models are used to transform the segmentation problem into PDE [13].

\section{LITERATURE SURVEY}

T. Rajesh et al., 2013[1] this research depicts all those medical images as input for the process, whose features will be extracted from particular images based on Rough Set Theory. Then these images will be processed under Feed Forward Neural Network classifier. This methodology in all consist of two particular functions in which the first function highlight the difference between abnormal and normal types of conduct where as the other one describes the classification of abnormality as malignant or benign.

A. Islam et al., 2013 [2] this research elaborate a stochastic model, which is very useful By using this model we can characterize the tumor texture in brain MRI.

E. E. Ulku et al., 2013 [3] represents the brain tumor detection using Computer-aided detection (CAD) system. This system is based on morphological image processing $\&$ histogram equalization technique, where the last stage of computer aided detection system i.e. classification and the classification algorithms in the Rapid Miner program is tested \& the comparison of all algorithm is done to show CAD system accuracy.

J. Vijay et al., 2013 [5] describes the brain tumor detection by using the technique called K-means clustering. The automatic segmentation of brain tumor is done to extract the tumor tissues from the brain.

Ishita Maiti et al., 2012[6] introduced a new method to detect brain tumor as watershed method in which initial RGB image gets converted in HSV colour image. This HSV image separates into three region as saturation, hue and intensity and to each region contrast enhancement watershed algorithm is applied and for output canny edge detection is used. This combined effect of all 3 images gives the final brain tumor segmented images.

\section{PROBLEM STATEMENT}

As per literature survey the previous method for this research was to segment the tumor region from the medical images by using the various types of segmentation methods and registration method, Those have been used to provide the accurate results but if the extracted region will be viewed as a $3 \mathrm{D}$ model it will provide better results. Area of extracted tumor and pixel difference value can be reduced more by using the proposed methodology. To show the exact part of tumor with area as well as pixel difference in 3D model the proposed system presents an extension to an existing segmentation algorithm. This research will modify the deformable registration of pre-operative and postrecurrence brain MR scans with biography based neural clustering (BBNC) by applying the Genetic algorithm. This new method handles various types of spaceoccupying, pathology, mass tumors. The iterating changes like varying details due to contrast and noncontrast imaging, noisy environment low quality resonance, sampling effects are also handle by this technique.

\section{OBJECTIVES}

- To select the previous output for recognising the initial values i.e. gene values, propagation values and duplicating gene detection for making clusters.

- To select the cluster, this will be processed for further analysis by applying the particular no. of iterations.

- To calculate the area, time and pixel difference. 
- The modelling of the extracted area using $3 \mathrm{~d}$ surface view will be done.

\section{METHODOLOGY USED FOR PROPOSED RESEARCH}

\section{BBNC with genetic processing}

BBNC stand for biography based neural clustering, is a method of clustering based on biography of medical images. Clustering can be done by using the multiple features (biography) of MRI or CT scans for differentiate a particular cluster consisting of healthy tissues or we can say in which lie the of tumor area. There are methods called neural clustering and genetic algorithms which are used in this research are below:-

\subsubsection{Neural clustering}

Clustering basically is a fundamental concept to analysis the data. It is used for pattern recognition, feature extraction and for image segmentation. In this research the clustering identifies some inherent structures present in the tumor region with the help of artificial neural network and then can make the multiple clusters basis on the biography of the medical images. This type clustering is known as neural clustering.

\subsubsection{Genetic algorithm}

The Genetic algorithm (GA) is a method for global research. Genetic algorithm is used for natural biological evolution. Genetic algorithm is operates on the population of potential solution by applying the principle of survival of fittest, which is further used to find out the better solutions. In genetic processing the each generation take place a new set of approximation solution that can be created by the process of selecting individuals' level of fitness accordingly. The uses of operators in the problem domain are borrowed from the natural Genetic process. This process further leads to the evolution of individuals and is also suited for their environment than the individuals. They were created from natural adaptation. The Genetic algorithm solves the problem of optimization by mimicking the principles of biological evolution. The modification of the population of individuals can be done by using the rules for gene combinations. The Genetic algorithm can also improve your chances of finding a global solution. It enables the researchers to solve bound constrained, unconstrained and optimization problems. It does not require the functions to be differentiable or continuous functions.

Segmentation of brain is supposed to be done by using Genetic algorithm. Following would be the steps for segmentation:

1. Acquire the medical image (MRI, CT) to be used for pre-processing.
2. Segmentation will be implemented on the acquired image by applying Genetic processing.

A. The Genetic algorithm will be initiated by creating a random and initial population.

B. Then the algorithm will creates a sequence of new populations, where at each step the algorithm will uses the individuals in the current generation to create the next population.

To create the new population, the algorithm performs the following steps:

a) Scores each member of the current population by computing its fitness value.

b) Scales the raw fitness scores to convert them into a more usable range of values.

c) Selects members known as parents according to their fitness.

d) Some individuals in the current population that have lower fitness value are chosen as Elite and these elite individuals are passed to the next population.

e) Produces children from the parents. Children are produced either by making random changes to a single parent are called mutation or by combining the vector entries of a pair of parents is called crossover.

f) Replaces the current population with the children to form the next generation.

C. The algorithm will be stop, when one of the stopping criteria is met. See Stopping Conditions for the Algorithm.

\section{RESULTS AND DISCUSSION}

The tumor region will be extracted from the MRI by using the genetic algorithm and neural clustering. Important steps to be performed are the genetic processing on the selected clusters, which further will be used to process on chromosome values based on images properties. Region of interest is also very important step which made the genetic processing easy and time saving. After performing the steps to enhance the quality of image the genetic processing will be initiate to make clusters. Than the selected cluster will be used to finishing the requirements can be viewed as 3D model. Results have been shown by base and proposed model are given below:-

The proposed methodology biography based neural clustering with genetic processing

The proposed methodology biography based neural clustering with genetic processing is very useful and time consuming technique to segment the tumor region from the medical images accurately. The results after processing the particular images from the data set of MRI or CT scans are given below. First of all, in the proposed methodology after getting the image from the dataset the noise from the image will be removed by applying the pre-processing, wavelet filter and thresholding methods. The denoised image will be processed for initiation of 
genetic process over the features of images or biography of images to make the clusters with the help of neural clustering. The selected clusters further will be processed by level set method of genetic by applying 100 iterations over the both proposed and previous methods. The extracted shape of tumor further will be shows in 3 Dimensional models which will help the diagnostics to find the exact area and time optimization. The results for proposed methodology are given below in fig 1:-

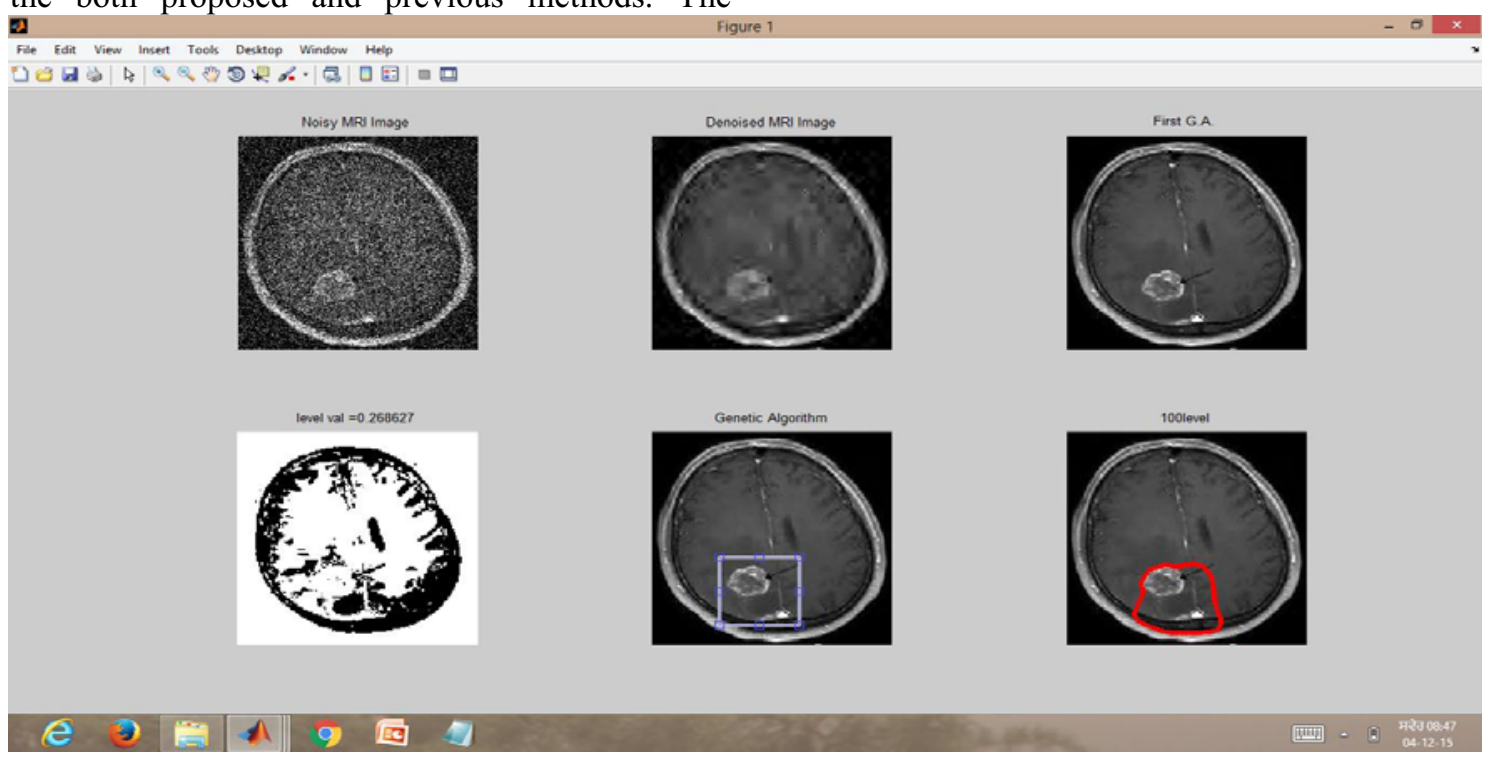

Figure 1. Processed medical images with proposed system

\section{Thresholding (5) genetic algorithm (6) shape extracted..}

\section{The previous methodology know as pre operative and} post reccurence registration

The previous methodology know as pre operative and post reccurence registration for medical images is also very useful technique to segment the tumor region from the medical images but it took more time to perform the same work by prosed technique. The results after processing the particular images from the dataset of MRI or CT scans are given below. First of all, in the previous methodology after getting the image from the dataset the noise from the image will be removed by applying the pre-processing, wavelet filter and thresholding methods. These process of segmentation will enhance the quality of
(1) Noisy MRI images (2) Denoised MRI images (3) First GA

images so that to segment out the tumor area easily. Upto this step the procedure of segmentation of previous method called PORTR and proposed method called BBNC is similar to each other. In previous method the denoised image will be processed for Pre operative registration. This method was for deformable registration of pre operative and post reccurence brain MRI scans for tumor patients. The selected clusters further will be processed under level set method by applying the 100 iterations over the both proposed and previous methods. The extracted shape of tumor further will be processed under $3 \mathrm{D}$ model to compare the results with proposed methodolgy.The detail of processesd PORTR with results are given below in fig 2:-

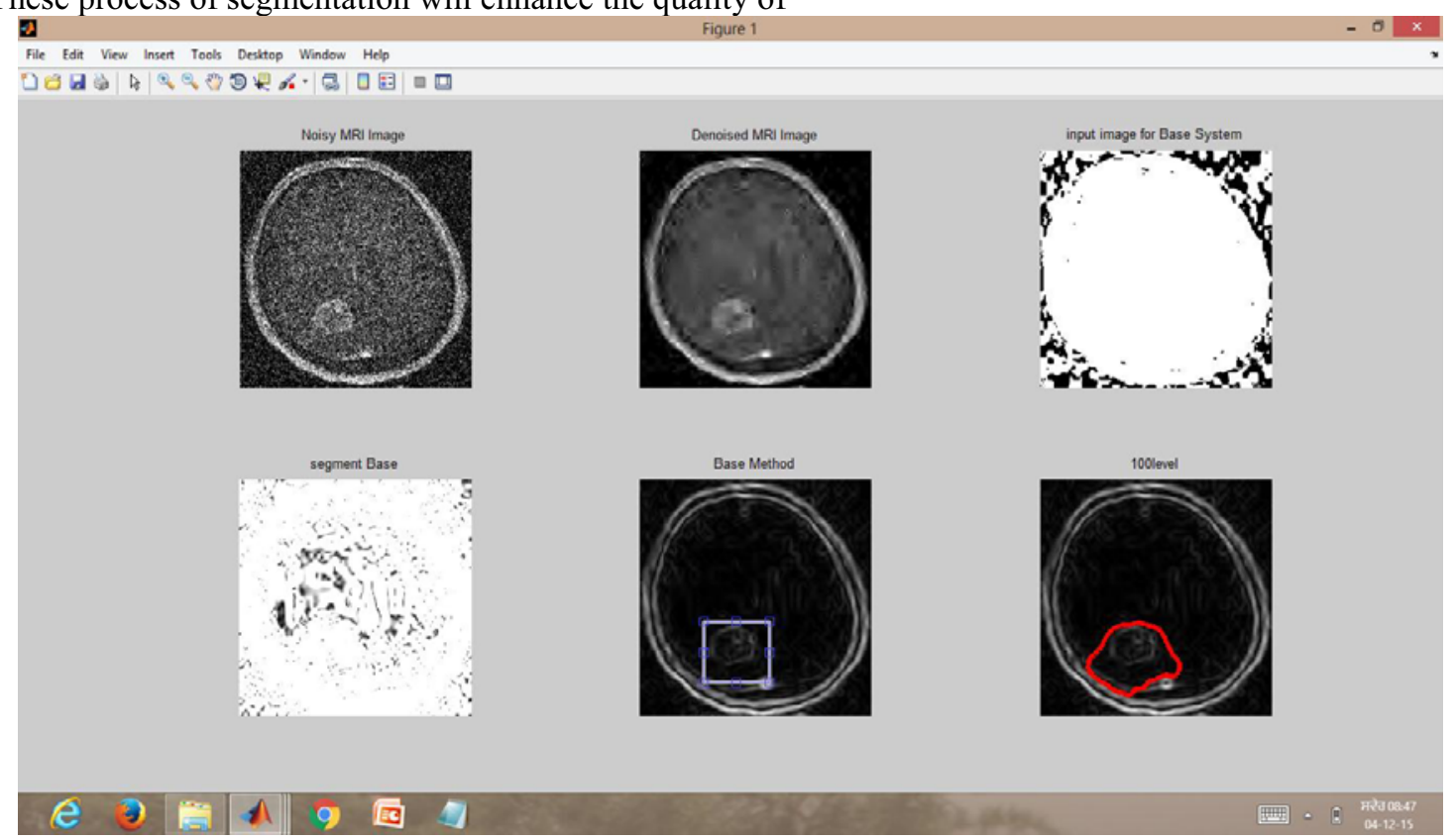


Figure 2. Processed medical images with previous approach (1) Noisy MRI images (2) Denoised MRI images (3) I/P image for PORTR (4) Segment PORTR (5) POTAR (6) shape extracted.

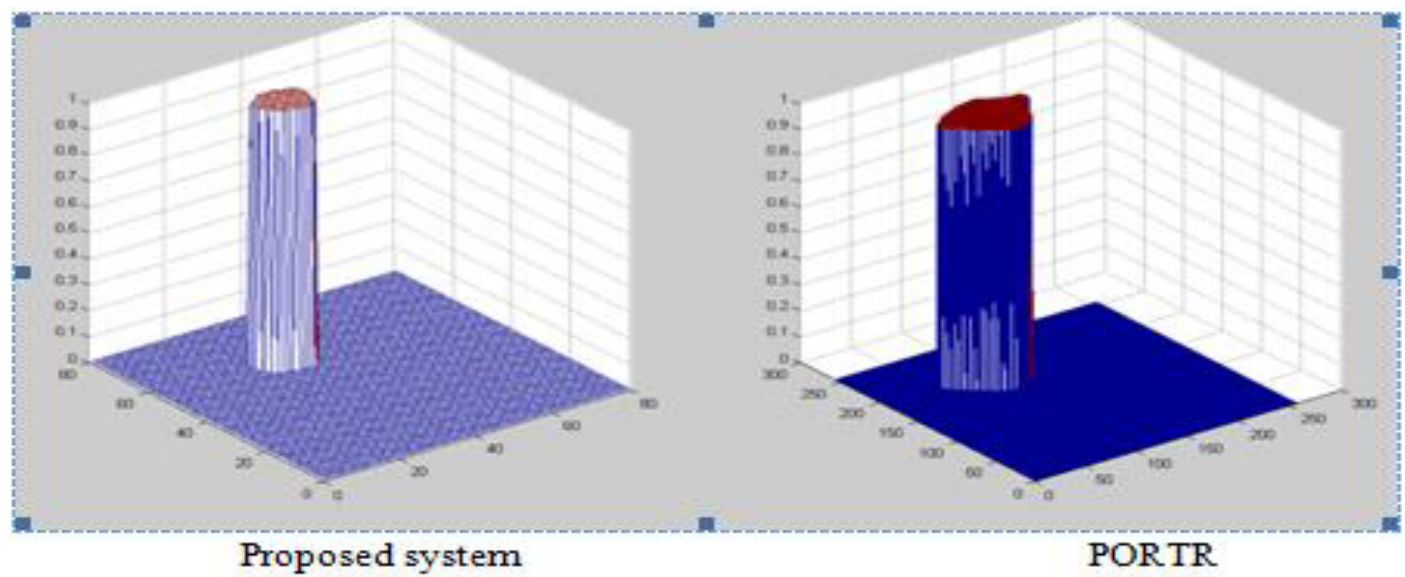

Figure 3. View of extracted region by both the techniques in 3 Dimension

The extracted region from both the methods will be compared after setup the level for 100 genetic iterations which will help to calculate the time optimization by both the methods and extracted area in given no. of iterations. The main aim of this research is to show the tumor area as three dimensional model to check the accuracy of the task performed to place the tumor area. So , the extracted region from both the techniques is viewed as 3 dimensional model which is in fig 3.

The extracted region of tumor in $3 \mathrm{D}$ shape tells us the accuracy of previous and proposed methods.Because the exacts part of tumor area extracted by applying the same no. of iterations on both the techniques provides different results. The values for all the parametors are given below in table 1.

Table 1. Shows the output previous and proposed system on the basis of area, iterations, pixel difference and time consumption margins.

\begin{tabular}{|l|l|l|l|l|}
\hline Results & Area & $\begin{array}{l}\text { No. of } \\
\text { Iteratio } \\
\mathrm{n}\end{array}$ & $\begin{array}{l}\text { Pixel } \\
\text { Differenc } \\
\mathrm{e}\end{array}$ & Time \\
\hline Base & $\begin{array}{l}105.5 \\
5\end{array}$ & 100 & 10555 & $\begin{array}{l}40.711 \\
2\end{array}$ \\
\hline $\begin{array}{l}\text { Propose } \\
\mathrm{d}\end{array}$ & 42.88 & 100 & 4288 & $\begin{array}{l}27.802 \\
2\end{array}$ \\
\hline
\end{tabular}

\section{CONCLUSION}

The proposed work concentrates on the division of the tumor tissue on the basis of neural clustering with genetic processing. In the Medical images (MRI and CT scans) the position of effected part can be visualize by expert easily but to extract the actual part of tumor like area and time optimization there is need to follow some software tools. This new technique will help the diagnostics as a tool to find the tumor area accurately. As previous method was also performing the same division of tissues but the proposed method improve the general precision by partitioning the exceedingly unprotected part and rejecting the strong tissue. This division is then finished up by a Neural Learning division structure. In view of base method the last division of proposed one ate up less time when it appeared differently in comparison of both methods. This shows the healthiness of the proposed structure over the base approach.

\section{REFERENCES}

1. T. Rajesh, R. Suja Mani Malar, (2013) "Rough Set Theory and Feed Forward Neural", international conference on advance nanomaterials and emerging engineering technologies (ICANMEET), pp 240-244.

2. Atiq Islam, Syed M. S. Reza, and Khan M. Iftekharuddin,(2013) "Multifractal Texture Estimation for Detection and Segmentation of Brain Tumors" IEEE Transactions On Biomedical Engineering, Vol. 60, No. 11, pp 3204-3215.

3. Eyup Emre Ulku, Ali Yilmaz Camurcu, (2013) "Computer Aided Brain Tumor Detection With Histogram Equalization And Morphological Image Processing Techniques", ICECCO, pp 48-51.

4. P Dvorak, W Kropatsch, and K Bartusek ,(2013) "Automatic Detection of Brain Tumors in MR Images", Telecommunication and Signal Processing, pp 577-5802. 5. J. Vijay, J. Subhashini,(2013) "An Efficient Brain Tumor Detection Methodology using k-means clustering algorithm" International conference on Communication and Signal Processing, pp 653-657.

6. I Maiti , Dr. Monisha Chakraborty, (2012) ,“A New Method for Brain Tumor Segmentation Based on Watershed and Edge Detection Algorithms in HSV Colour Model", Computing and communication systems (NCCCS), pp 1-5.

7. Natarajan P, Krishnan.N, Natasha Sandeep Kenkre, Shraiya Nancy, Bhuvanesh Pratap Singh, "Tumor Detection using threshold operation in MRI Brain Images", Computational intelligence and computing research (ICCIC) IEEE International conference, pp 1-4. 
8. Azian Azamimi Abdullah, Bu Sze Chize,(2012) Yoshifumi Nishio, "Implementation of An Improved Cellular Neural Network Algorithm For Brain Tumor Detection", International Conference on Biomedical Engineering (ICOBE), pp 611-615.

9. China, Phooi Yee Lau, Frank C. T. Voon, and Shinji Ozawa, (2009)“The detection and visualization of brain tumors on T2-weighted MRI images using multiparameter feature blocks", Engineering in Medicine and Biology 27th Annual Conference, pp 5104-5107.

10. P. Singh, (2013) "A new approach to image segmentation", International Journal of Advanced Research in Computer Science and Software Engineering, Vol. 3, No. 4..

11. H. G. Kaganami and Z. Beij,(2009) "Region based detection versus edge detection," IEEE Transactions on Intelligent Information Hiding and Multimedia Signal Processing, pp. 1217-1221.

12. X. iang, R. Zhang, and S. Nie,(2009) "Image segmentation based on PDEs model: A survey", in Proc. 3rd International Conference on Bioinformatics and Biomedical Engineering, pp. 1-4.

13. B. C. Wei, R. Mandava, (2010)“Multiobjective optimization Approaches in Image Segmentation- the Direction and Challenges", ICSRS Publication.

14. W.D. Heiss, P. Raab, and H. Lanfermann, (2011)"Multimodality assessment of brain tumors and tumor recurrence," J. Nucl. Med., Vol. 52, No. 10, pp. 1585-1600.

15. Dongjin Kwon, (2014) "PORTR: Pre-Operative and Post-Recurrence Brain Tumor Registration" ieee transactions on medical imaging", Vol. 33, No. 3, pp. 0278-0062. 